\title{
Viscoelastic Properties of Concentrated Suspensions
}

\author{
F. W. A. M. SCHREUDER, A. J. G. VAN DIEMEN, AND H. N. STEIN \\ Laboratory of Colloid Chemistry, Eindhoven University of Technology, \\ P.O. Box 513, 5600 MB Eindhoven, The Netherlands
}

Received January 31, 1985; accepted July 10, 1985

\begin{abstract}
The deformation observed during oscillatory shear of concentrated suspensions indicates the alternate occurrence of two structures: the first at small deformations gives rise to approximately linear viscoelastic behavior, while the second is a virtually undeformable structure formed at large deformations. The behavior at small deformation is characterized by a dynamic viscosity which at low frequencies is inversely proportional to the frequency: at higher frequencies the viscosity levels off. This transition is ascribed to sheet formation by the suspended particles during shear in the low frequency region; in the high frequency region sheet formation is less developed. In accordance with this interpretation is the deformation at which the undeformable structure originates, as a function of frequency. The origin of this structure is attributed to the following: once the shear leads to an increase in solid content in a part of the system, the apparent viscosity there rises which causes an additional increase in solid content. This finally leads to a blocking of further deformation. (c) 1986 Academic Press, Inc.
\end{abstract}

\section{INTRODUCTION}

Silanization of the surface of an inorganic solid appears to change profoundly the rheological properties of its dispersions in organic liquids (1-8). The effect is related to the tendency of the solid particles to coagulate and thus depends on the polarity of the medium (2).

In dioctylphthalate (DOP) as a medium (apolar), no difference in steady flow rheological behavior could be observed between suspensions of unsilanized glass beads (hydrophilic) on the one hand, and suspensions of silanized glass beads (hydrophobic) on the other (6). The relative viscosities are identical for suspensions of hydrophilic and hydrophobic glass beads in DOP, and for suspensions of hydrophilic glass beads in glycerol, a polar system. Absence of difference was also found for $\tau_{0} / \eta_{0}$. Here $\tau_{0}$ is a constant which describes the steady flow rheology at high shear rates in suspensions of high solid content according to

$$
\tau=\tau_{0}+\eta_{\mathrm{PL}} \dot{\gamma}
$$

with $\tau=$ shear stress, $\dot{\gamma}=$ shear rate, and $\eta_{\mathrm{PL}}$ $=\mathrm{a}$ constant at given temperature, pressure, and composition; while $\eta_{0}$ is the viscosity of the medium. Thus in dioctylphtalate no coagulation can be observed from the rheological properties. This interpretation was confirmed by sedimentation rate measurements.

Nevertheless, the rheological behavior described by Eq. [1] is shear-thinning. Generally, shear-thinning in suspensions is ascribed to the disruption of aggregates with increasing shear rate (see, e.g., Refs. 9 and 10). However, how can shear-thinning be understood when the rheological behavior is not influenced by coagulation?

Two of the present authors, in a previous paper (6), suggested that sheet formation by particles during shear (11-13) is held responsible for the shear-thinning character. On calculating the energy dissipation per unit of volume and time in simple shear by means of

$$
\dot{\epsilon}=\tau * \dot{\gamma},
$$

one arrives at the conclusion that it is composed of two parts, 


$$
\begin{aligned}
& \dot{\epsilon}_{1}=\eta_{\mathrm{PL}} * \dot{\gamma}^{2} \\
& \dot{\epsilon}_{2}=\tau_{0} * \dot{\gamma} .
\end{aligned}
$$

In concentrated suspensions the energy dissipation during flow is supposed to be predominantly due to encounters between the suspended particles, and the number of encounters is thought to be proportional to $\dot{\gamma}$. Then $\dot{\epsilon}_{1}$ refers to processes in which the energy dissipation per encounter is proportional to the relative velocity of the particles concerned. This is to be expected on the basis of sheet formation during shear, if a collision between particles belonging to two successive layers is accompanied by momentum transfer.

On the same reasoning, however, $\dot{\epsilon}_{2}$ would correspond to processes, in which the energy dissipation per encounter is independent of the relative velocity of the particles involved. The explanation for the case of dilute suspensions, viz., that the colliding particles are flocs whose size decreases with increasing $\dot{\gamma}$ (14), cannot be applied to noncoagulating suspensions of high solid content. Neither can the hypothesis be defended that the energy dissipation concerned is connected with pair deformation and separation (6).

In our previous paper (6), the hypothesis was developed that during a rapid collision fewer neighbor spheres are entrained than during a slow one: during a rapid collision, a large fraction of the local stress is caught elastically, whereas during a slow one the local stress causes more viscous motion of adjacent spheres. If the energy dissipation is related primarily to the work necessary for moving a particle through the suspension, then an energy dissipation term according to Eq. [4] might be expected.

We started the present investigation in order to test the hypothesis expressed in the preceding paragraph. If the hypothesis is true, concentrated suspensions should show viscoelasticity even in the absence of rheologically perceptable coagulation. Thus we investigated the viscoelastic behavior of the suspensions concerned (of spherical glass beads, either hydrophilic or phydrophobic, in DOP).

For investigating viscoelasticity we used oscillatory torsion. An advantage of this method is that suspensions of higher solid content can be examined than by steady flow; thus we are able to check whether the absence of influence on the rheological properties of a hydrophobic layer on the glass particles in DOP extends to a solid volume fraction bordering on close packing of the suspended particles.

Among the previous investigations of concentrated suspensions, we focus attention on those concerning suspensions in Newtonian liquids. Akers and Machin (15), on subjecting flocculated suspensions of $\mathrm{CaCO}_{3}$ in aqueous polyacrylamide solutions to a sinusoidal strain, found that the stress does not have a sinusoidal shape, but is topped off as soon as it surpasses a certain yield value. This yield value decreases with increasing number of cycles. Simultaneously, both viscous modulus $\left(G^{\prime \prime}\right)$ and elastic modulus $\left(G^{\prime}\right)$ decrease, the latter (relatively) more rapidly than the former. On the other hand, Strivens (16-17) investigating noncoagulating, sterically stabilized suspensions, did not report time-dependencies of rheological parameters. He found a transition from a high viscosity, low $G^{\prime}$ region at low frequencies to a low viscosity, high $G^{\prime}$ region at high frequencies. The transition was found at an angular frequency of about $1 \mathrm{rad} / \mathrm{sec}$; it was ascribed to ordering of the suspended particles into sheets under shear in the low frequency region. In the high frequency region, this ordering is less developed because there the hydrodynamic forces do not persist long enough in one direction; thus here Brownian motion was supposed to predominate.

We investigated suspensions of rather large spherical particles $(45-63 \mu \mathrm{m})$ in rather viscous media ( 80 and $150 \mathrm{mPasec}$ ), such as to eliminate any influence of Brownian motion. In such suspensions, the time necessary for diffusion over a distance equal to the particle radius is of the order 40-80 days, thus much longer than the time scale of the experiments. 


\section{EXPERIMENTAL}

\section{Materials}

Glass Ballotini. Ex Tamson (Zoetermeer), specific mass $(293 \mathrm{~K}) 2750 \mathrm{~kg} \mathrm{~m}^{-3}$. The fraction used was separated by dry sieving. Part of this sample was made hydrophobic by the method described by Kao et al. (2) (see also Ref. 6). The size distribution, as found by sedimentation in a $50 \% \mathrm{~m} / \mathrm{m}$ aqueous sucrose solution in a Micromeritics Type 5000D Sedigraph, was as follows: $20 \% \mathrm{~m} / \mathrm{m}$ smaller than $45 \mu \mathrm{m} ; 50 \% \mathrm{~m} / \mathrm{m}$ smaller than $54 \mu \mathrm{m} ; 80 \%$ $\mathrm{m} / \mathrm{m}$ smaller than $65 \mu \mathrm{m}$.

Dioctylphthalate. Di-2-ethylhexyl phthalate, ex Hoechst, of technical purity; viscosity (293 K) 79.3 mPasec.

Glycerol plus water mixture. "Glycerol $(87 \%)$," ex Merck, pro analysi, viscosity $(293 \mathrm{~K}) 150.5 \mathrm{mPasec}$; refractive index $n_{\mathrm{D}}^{25}$ $=1.4533$; from the refractive index, the water content was estimated to be $13.1 \% \mathrm{~m} / \mathrm{m}$.

\section{Apparatus}

An apparatus for oscillatory torsion, supplied by TNO Delft, was employed. Its essential features have been described by den Otter (18), Duiser (19), and te Nijnenhuis (20). In this apparatus, the sample is contained in the space between two coaxial cylinders. The inner cylinder is centered by two torsion wires. One is fixed to the bottom of the apparatus and to the bottom of the inner cylinder; the other is fixed to its top and to a point subject to a sinusoidal torsional motion $\alpha_{\mathrm{a}}=\alpha_{\mathrm{a} 0} \sin \omega t$, with $\alpha_{\mathrm{a} 0} \sim 0.1 \mathrm{rad}$ and $\omega=$ angular frequency. The latter will be called "drive deformation," while the oscillation of the inner cylinder is indicated as "response deformation."

The angular deflection from the ends of the upper torsion wire, $\alpha_{\mathrm{a}}$ and $\alpha$ (=that pertaining to the inner cylinder) were followed by light beams reflected from mirrors onto a scale at a distance of $2.0 \mathrm{~m}$.
The positions of the reflected light beams on the scale can be followed by eye. At low frequencies the position of the individual spots can be followed as a function of time; at higher frequencies, only the maximum deformation $\left(\alpha_{\max }\right)$ can be read directly. A phase angle difference $(\phi)$ can be observed between the respective motions.

Electronically the time was measured between the passage of the reflected light beams over photosensitive transistors, placed at selected positions on the scale; a Hewlett-Packard type 5328 A counter was employed. From the time measurements $\alpha_{\mathrm{a} 0}$ and $\alpha_{0}$ could be calculated; $\alpha_{\mathrm{a} 0}=$ the amplitude of the drive deformation; $\alpha_{0}=$ the amplitude of the response deformation as calculated from the time dependence at small deformations, supposing the response to be sinusoidal. $\alpha_{0}$ will, in general, not be equal to $\alpha_{\max }$ (see Results). The phase angle can be derived in a similar way.

The torsion wires employed had the following constants: lower torsion wire, $5.06 * 10^{-5}$ $\mathrm{Nm}$; upper torsion wire, $1.28 * 10^{-3} \mathrm{Nm}$.

\section{Procedure}

Pastes were mixed by hand. A solid volume fraction of 0.63 was used throughout. Filling of the rheometer was effected by squeezing the paste from a silicon rubber tube into the apparatus.

Squeezing had to be performed carefully and slowly, in order to avoid having the flow became blocked. Before measurements, the paste in the apparatus was subjected to an oscillation at $2500 \mathrm{rpm}$ for $10 \mathrm{~min}$ followed by 10 min rest.

This method of sample preparation resulted in a reasonably, though not exactly, reproducible state of the sample, i.e., reproducible packing of the suspended particles as apparent from the scatter of the rheological properties (see Results). We intend to return to the relation between sample preparation method and rheological properties in the future. 


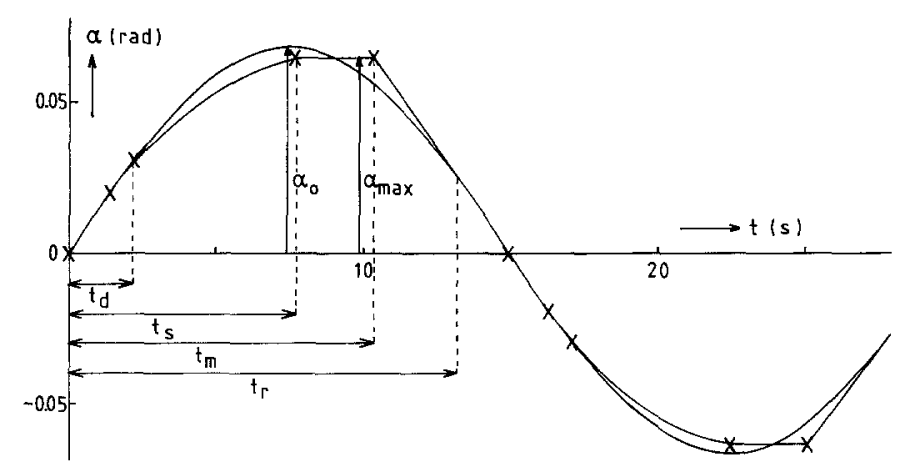

FIG. 1. The response deformation $(\alpha)$ as a function of time. $\times$, Actual; - , sinusoidal, as calculated from the time at low $\alpha$ values.

\section{RESULTS}

\section{A. Form of the Response Deformation Curve}

Figure 1 shows the form of the response deformation, as observed by eye at low frequencies, as a function of time. At low values, the points are situated to a very good approximation on a sinusoidal curve:

$$
\alpha=\alpha_{0} \sin (\omega t)
$$

However, for larger values of $\alpha$ differences between the observed values and those predicted by [5], with $\alpha_{0}$ calculated from small $\alpha$ values, are found. At some time $t_{\mathrm{s}}$ the deformation stops entirely (as far as can be observed with the apparatus) at a value $\alpha_{\max }$. However, the halt in deformation finishes at a time $t_{\mathrm{m}}$ where motion starts again. Both $t_{\mathrm{s}}$ and $t_{\mathrm{m}}$ are observed as sharp breaks, which can be recorded by a stopwatch, as long as the angular frequency $\omega$ is low enough to follow the individual reflected beams by eye.

At higher frequencies this is no longer possible. Nevertheless, $\alpha_{\max }$ can be observed and differences between $\alpha_{0}$ and $\alpha_{\max }$ are found similar to those observed at lower frequencies. Again, $\alpha_{0}$ is calculated from the time at which the reflection light beam passes a photosensitive transistor placed at $\alpha=0.01 \mathrm{rad}$, on the assumption that at this low deformation Eq. [5] is obeyed.

The form of the $\alpha$ vs $t$ curve indicates that during oscillatory shear alternatively two structures are formed: at low deformation a structure showing linear viscoelastic behavior to a good approximation; at large deformation a practically undeformable structure; with transition regions in between (for $t_{\mathrm{d}}<t<t_{\mathrm{s}}$ and $t_{\mathrm{m}}<t<t_{\mathrm{r}}$ in Fig. 1).

We shall first describe some experimental facts before interpreting the data.

\section{B. Behavior at Small Deformations}

From $\alpha_{0}$ (at a given amplitude of the drive deformation) and $\phi$ the moduli $G^{\prime}$ and $G^{\prime \prime}$ can be calculated according to standard equations (20). Both $G^{\prime}$ and $G^{\prime \prime}$ depend on the number of cycles passed through: after an initial period during which clearly a stationary state is established, $G^{\prime}$ and $G^{\prime \prime}$ increase with increasing time (Figs. 2 and 3). Quite generally $\log G^{\prime}$ increases more rapidly than $\log G^{\prime \prime}$; this is also expressed in Fig. 4, showing $\log \operatorname{tg}(\delta)$ as a function of time $\left(\operatorname{tg}(\delta)=G^{\prime \prime} / G^{\prime} ; \delta=\right.$ loss angle).

Figure 5 shows $\log G^{\prime}$ and $\log G^{\prime \prime}$ measured after a definite time $(t=2 \mathrm{~min})$, as a function of the angular frequency.

The decrease of $\log \operatorname{tg}(\delta)$ with increasing time, thus with increasing number of cycles passed through (Fig. 4), corresponds with a progressively more elastic, less dissipative character of the paste when mobile. The dynamic viscosity

$$
\eta^{\prime}=G^{\prime \prime} / \omega
$$




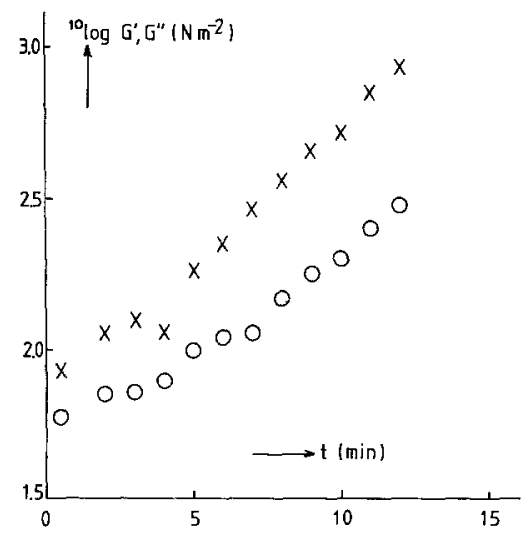

Fig. 2. $\log G^{\prime}$ and $\log G^{\prime \prime}$ as a function of time; untreated glass in DOP, $\omega=0.6576 \mathrm{rad} \mathrm{sec}^{-1} . \times, \log G^{\prime} ; O, \log G^{\prime \prime}$.

to be sure, increases with increasing number of cycles passed through; but, since $\log G^{\prime}$ increases more rapidly than $\log G^{\prime \prime}$ the dissipative character of the paste can be said to decrease notwithstanding the viscosity increase.

\section{Behavior at Large Deformations}

The apparatus permits only one characteristic feature of the paste in its undeformable state to be measured: $\alpha_{\max }$. Figure 6 shows $\alpha_{\max }$ as a function of time for some frequencies.

$\alpha_{\max }$ decreases with increasing time. The initial value of $\alpha_{\max }$ increases with decreasing

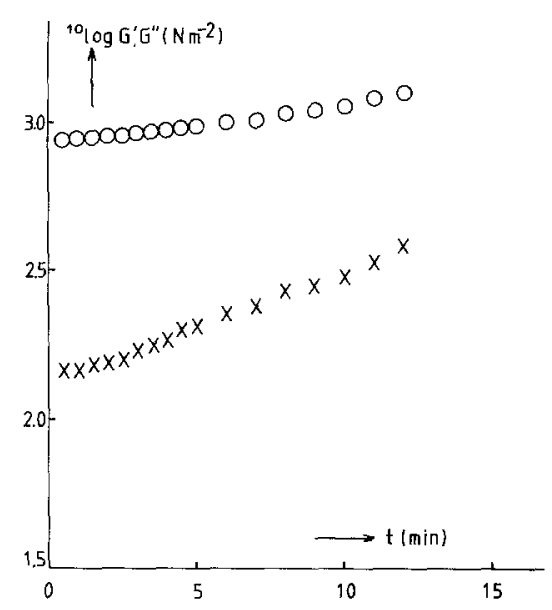

FIG. 3. As Fig. 2, $w=209.14 \mathrm{rad} \mathrm{sec}^{-1}$.

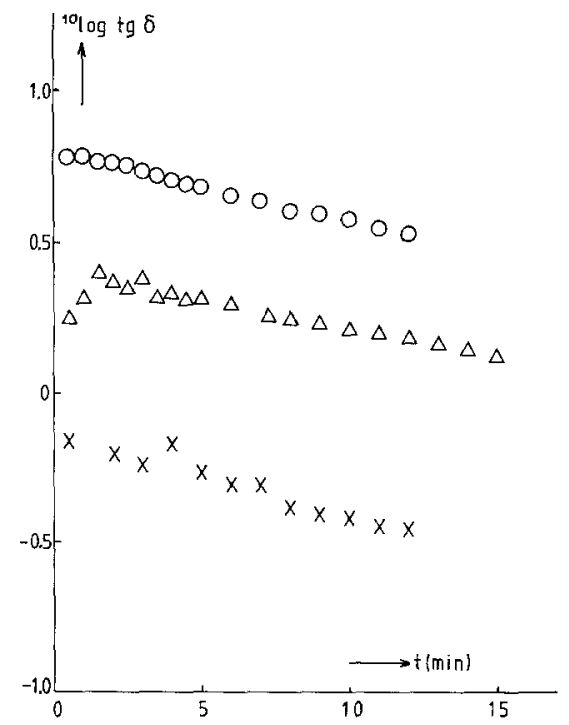

FIG. 4. Log $\operatorname{tg} \delta$ as a function of time for suspensions of untreated glass in DOP. $\times, \omega=0.6576 \mathrm{rad} \mathrm{sec}^{-1} ; \Delta, \omega$ $=24.19 \mathrm{rad} \mathrm{sec}^{-1} ; O, \omega=209.44 \mathrm{rad} \mathrm{sec}{ }^{-1}$.

angular frequency. At low $\omega, \alpha_{\max }$ decreases more rapidly with increasing time than at higher frequencies.

\section{DISCUSSION}

The most striking feature of the experimental data is the attainment of a limit to the deformation $\alpha_{\max }$ indicating the formation of an

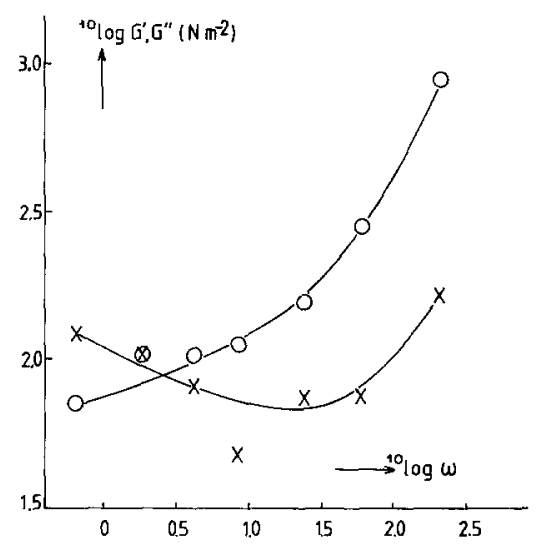

FIG. 5. $\log G^{\prime}$ and $\log G^{\prime \prime}$, measured after $2 \mathrm{~min}$, as a function of frequency. Untreated glass in DOP. $\times, \log G^{\prime}$; O, $\log G^{\prime \prime}$. 


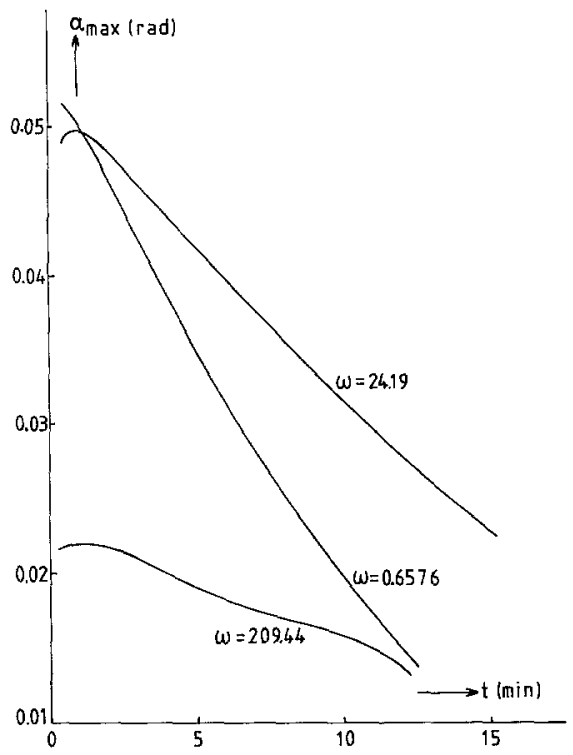

FIG. 6. $\alpha_{\max }$ as a function of time. Untreated glass in DOP.

undeformabie structure. The existence of this limit proves that structures in concentrated suspensions can support stresses elastically.

Clearly, the deformation leads to accumulation of the suspended particles, which can block further deformation. The same was experienced on filling the apparatus, if the squeezing of the paste into the space between the two coaxial cylinders was performed too fast; this effect occurred especially if the paste was pushed into its place by means of a piston. Similar observations have already been reported by Freundlich and Jones (21).

It is remarkable that there is not one general value of $\alpha_{\max }$ but that $\alpha_{\max }$ is dependent on both frequency and number of cycles passed through. In addition, there is a transition region between the viscoelastic and the undeformable states. The best way to account for these facts is that during deformation, the solid particles arraign themselves into a closepacked structure in a part of the system. There the apparent viscosity increases sharply, which leads to an additional accumulation of solid particles entailing an even further increased apparent viscosity. Finally, such a large part of the system is occupied by particles in close packing that further deformation (other than purely elastic compression) becomes impossible.

On subjecting the system in its undeformable state, in a later stage of a cycle, to a tensile stress, the structure is broken down such as to permit flow. However, the undeformable structure is not broken down completely, as evidenced by the dependence of $\alpha_{\max }$ and of $\log G^{\prime}$ and $\log G^{\prime \prime}$ on the number of cycles passed through.

The demolition of the undeformable structure is more pronounced at higher frequencies than at lower ones (cf. Fig. 6). Three reasons could be held responsible for this:

(a) the higher shear rates at higher frequencies;

(b) the more rapid transition from compressive to tensile stresses at higher frequencies, entailing larger tensile stresses to operate on the undeformable structure because the latter needs some time before yielding to tensile stresses;

(c) a less pervasive character of the undeformable structure at high frequencies, because here the particles cannot adjust themselves to shear to the same extent as at low frequencies, before deformation is blocked.

The third argument certainly is operative; it is confirmed by the initial dependence of $\alpha_{\max }$ on frequency. Sheet formation accompanying the transition of randomly packed particles into close packing (11) is certainly compatible with the results. Sheets permitting deformation in one direction can be employed for the reverse direction as long as the sheets remain aligned; but if they are diverted from the direction of movement they will obstruct deformation more strongly than particles in a random packing because within the sheets the number of nearest neighbors of a particle is high.

In order to investigate whether coagulation is important in these phenomena, we per- 

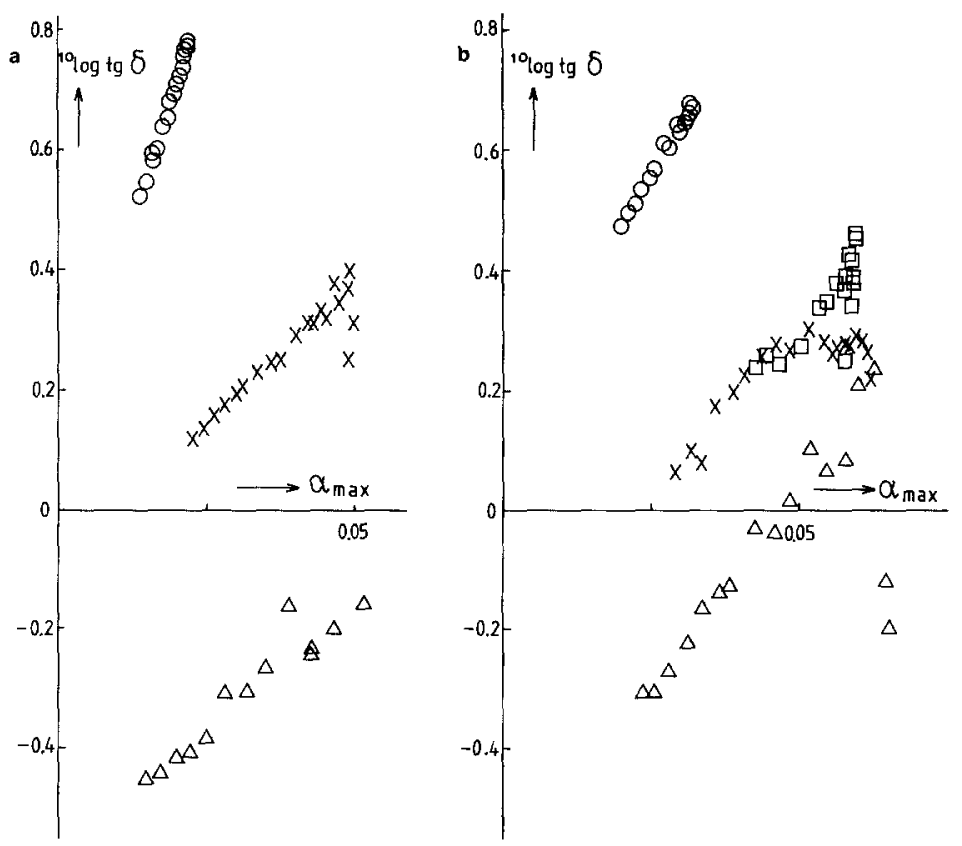

FIG. 7. Log tg $\delta$ vs $\alpha_{\text {max }}$ for various frequencies. (a) Untreated glass in DOP: $O, \omega=209.4 ; \times, \omega=24.19$; $\triangle, \omega=0.654 \mathrm{rad} \mathrm{sec}^{-1}$. (b) Silanized glass in DOP: $O, \omega=210.2 ; \times, \omega=9.43 ; \Delta, \omega=0.602 ; \square, \omega=28.6$ $\mathrm{rad} \sec ^{-1}$.

formed the same experiments with hydrophobic glass. Figure 7 compares $\log \operatorname{tg}(\delta)$ as a function of $\alpha_{\max }$ for suspensions of hydrophilic glass in DOP (Fig. 7a) and for suspensions of hydrophobic glass in the same medium (Fig. 7b). Apart from an initial period, both $\log \operatorname{tg}(\delta)$ and $\alpha_{\max }$ decrease with increasing number of cycles.

Though in quantitative respect there are differences between hydrophilic and hydrophobic glass suspensions, the broad features of Figs. $7 \mathrm{a}$ and $7 \mathrm{~b}$ are the same. Thus silanization influences neither the formation of an undeformable structure nor the elastic character of the system when mobile.

Similar results are found when comparing suspensions of hydrophilic and hydrophobic glass particles in a glycerol + water mixture (Figs. 8, 9, and 10). This is especially remarkable because in this medium, at lower solid contents, distinct differences are found between the two types of suspensions $(6,8)$. Thus we have to conclude that the phenomena are not influenced by coagulation to an extent leading to qualitative differences.

In order to discuss the consequences of these

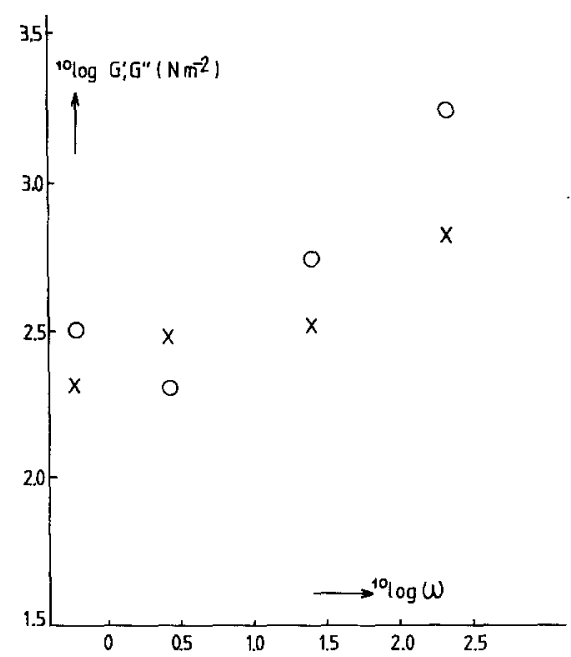

FIG. 8. $\log G^{\prime}$ and $\log G^{\prime \prime}$ as a function of frequency for suspensions in glycerol. Untreated glass, $t=2 \mathrm{~min}$. $\times$, $\log G^{\prime} ; O, \log G^{\prime \prime}$. 


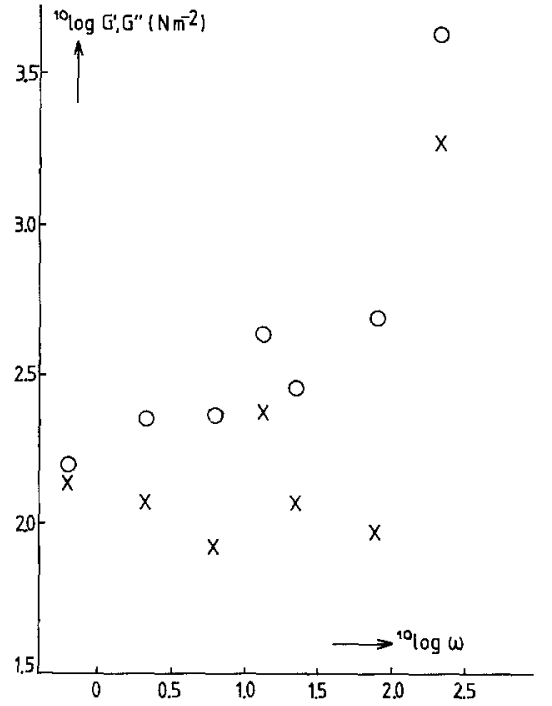

FIG. 9. $\log G^{\prime}$ and $\log G^{\prime \prime}$ as a function of frequency for suspensions in glycerol. Silanized glass; $t=2 \mathrm{~min}$. $\mathrm{X}$, $\log G^{\prime} ; \bigcirc, \log G^{\prime \prime}$.

phenomena for the energy dissipation in the material when mobile, we consider one complete cycle to be passed through in the linar viscoelastic structure without the undeformable structure being formed. The energy dissipation per unit volume during one cycle is given by

$$
\Delta E=\int_{\omega t=-\delta}^{\omega t=2 \pi-\delta} \tau \dot{\gamma} d t=\pi \gamma_{0}^{2} G^{\prime \prime},
$$

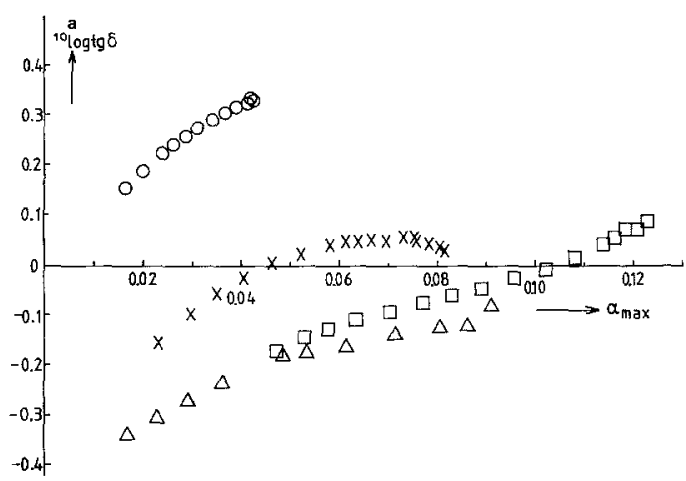

thus the energy dissipation per unit of volume and time becomes

$$
\dot{\epsilon}=(\omega / 2 \pi) * \Delta E=\frac{1}{2} \omega^{2} \gamma_{0}^{2} \eta^{\prime} .
$$

We define an average value of $\dot{\gamma}$ by

$$
\langle\dot{\gamma}\rangle=\frac{\int_{0}^{\pi} \dot{\gamma} d(\omega t+\delta)}{\int_{0}^{\pi} d(\omega t+\delta)}=\frac{2 \omega \gamma_{0}}{\pi}
$$

Thus,

$$
\dot{\epsilon}=\left(\pi^{2} / 8\right) \eta^{\prime}\langle\dot{\gamma}\rangle^{2} .
$$

For comparing $\dot{\epsilon}$ at different frequencies we consider a number of such oscillations at equal values of $\gamma_{0}$. Thus we obtain the best comparison with steady shear values at different shear rates, as investigated in our previous work $(6,8) .\langle\dot{\gamma}\rangle$ is then $\sim \omega$.

If $\eta^{\prime}$ is independent of $\omega, \dot{\epsilon}$ would be proportional to $\langle\dot{\gamma}\rangle^{2}$. This would mean that the energy dissipation per collision is proportional to $\langle\dot{\gamma}\rangle$, thus proportional to the relative velocity of two colliding particles. If, on the other hand, $\eta^{\prime}$ is proportional to $1 / \omega$, the energy dissipation per collision is independent of the relative velocity of colliding particles.

Experimentally, in the low frequency region $\eta^{\prime}$ is, in good approximation, inversely proportional to $\omega$ (Fig. 11). Here, where indications of sheet formation are found, the situa-

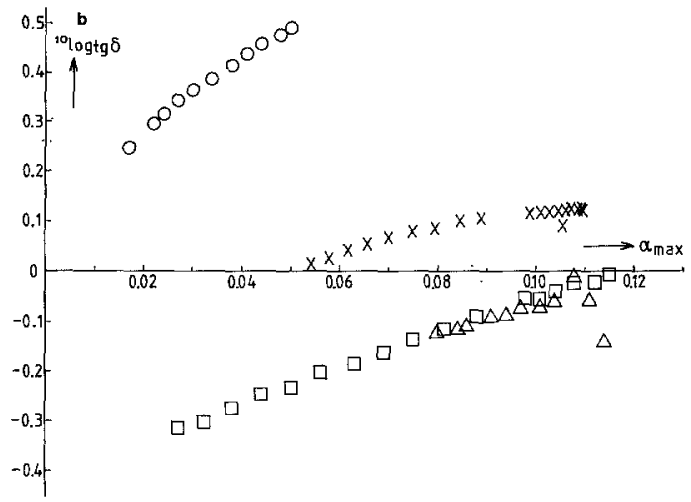

FIG. 10. Log $\operatorname{tg} \delta$ vs $\alpha_{\max }$ for various frequencies. (a) Untreated glass in glycerol; (b) silanized glass in glycerol. (a) $\triangle, \omega=0.634 \mathrm{rad} \mathrm{sec}-1, \square, \omega=2.09 \mathrm{rad} \mathrm{sec}^{-1}, x, \omega=21.78 \mathrm{rad} \sec ^{-1}, O, \omega=206.8 \mathrm{rad} \mathrm{sec} \mathrm{se}^{-1}$; (b) $\Delta, \omega=0.601 \mathrm{rad} \mathrm{sec}^{-1}, \square, \omega=2.61 \mathrm{rad} \mathrm{sec}-1, X, \omega=25.34 \mathrm{rad} \mathrm{sec}-1,0, \omega=210.9 \mathrm{rad} \mathrm{sec}^{-1}$. 


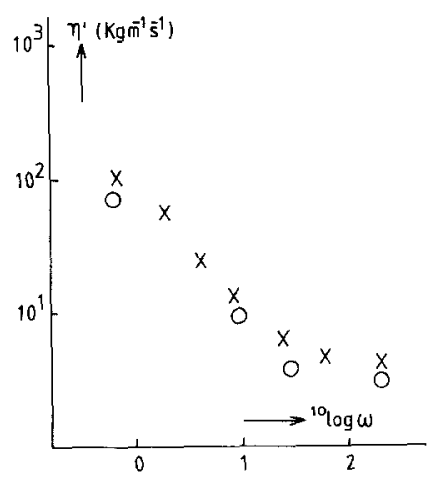

FIG. 11. $\eta^{\prime}$ as a function of frequency, for suspensions in DOP. $\times$, Untreated glass; $O$, silanized glass.

tion concerning energy dissipation is similar to that observed during steady flow of less concentrated suspensions (6). However, in the high frequency region, where sheet formation is less developed, $\eta^{\prime}$ levels off. In the frequency region where the experiments are compatible with sheet formation during shear, the energy dissipated per collision is independent of the relative velocity of the colliding particles; this is in agreement with the hypothesis previously offered. A strong or weak tendency to coagulation plays no important part in these phenomena.

\section{CONCLUSIONS}

Structures in concentrated suspensions formed by shear can support elastic stresses. This is independent of strong or weak tendency to coagulation.

During oscillatory shear two structures alternate: the first at small deformations gives rise to approximately linear viscoelastic behavior, while the second is a virtually undeformable structure formed at large deformation.

The experimental results can be explained by the following mechanism: At low frequencies sheet formation occurs during shear and the energy dissipated per collision between suspended particles is independent of the relative velocity of the particles. At high frequencies sheet formation is less developed and the energy dissipation per collision becomes proportional to the relative velocity of the colliding particles.

\section{REFERENCES}

1. Chapman, F. M., and Lee, T. S., Soc. Plast. Eng. J. 26(1), 37 (1970).

2. Kao, S. V., Nielsen, L. E., and Hill, Ch. T., J. Colloid Interface Sci. 53, 358 (1975).

3. Minagawa, N., and White, J. L., J. Appl. Polym. Sci. 20, 501 (1966).

4. Tanaka, H., and White, J. L., Polym. Eng. Sci. 20, 949 (1980).

5. Han, C. D., van der Weghe, T., Shete, P., and Haw, J. R., Polym. Eng. Sci. 21, 196 (1981).

6. van Diemen, A. J. G., and Stein, H. N., J. Colloid Interface Sci. 86, 318 (1982).

7. Suetsugu, Y., and White, J. L., J. Appl. Polym. Sci. 28, 1481 (1983).

8. van Diemen, A. J. G., Schreuder, F. W. A. M., and Stein, H. N., J. Colloid Interface Sci. 104, 87 (1985).

9. Firth, B. A., and Hunter, R. J., J. Colloid Interface Sci. 57, 248, 266 (1976).

10. Firth, B. A., J. Colloid Interface Sci. 57, 257 (1976).

11. Hoffman, R. L., Trans. Soc. Rheol. 16, 155 (1972).

12. Hoffman, R. L., J. Colloid Interface Sci. 46, 491 (1974).

13. Hoffman, R. L., Adv. Colloid Interface Sci. 17, 161 (1982).

14. Hunter, R. J., and Frayne, J., J. Colloid Interface Sci. 76, 107 (1980).

15. Akers, R. J., and Machin, G. P., Preprints, 2nd World Filtration Congress, 1979, p. 365.

16. Strivens, T. A., J. Colloid Interface Sci. 57, 476 (1976).

17. Strivens, T. A., Colloid \& Polym. Sci. 261, 74 (1983).

18. den Otter, J. L., Rheol. Acta 8, 355 (1969).

19. Duiser, J. A., "Het viscoelastische gedrag van twee polycarbonzuren in water," Ph.D. thesis, Leiden, 1965.

20. te Nijenhuis, K., "Dynamic Mechanical Studies on Thermo-Reversible Ageing Processes in Gels of Polyvinyl Chloride and Gelatin," Ph.D. thesis, Delft, 1979.

21. Freundlich, H., and Jones, A. D., J. Phys. Chem. 40, 1217 (1936). 